\title{
Interaction of relativistic short proton bunches with space charge limited electron clouds
}

\author{
F. B. Petrov, ${ }^{1, *}$ O. Boine-Frankenheim, ${ }^{1,2}$ and O. S. Haas ${ }^{1}$ \\ ${ }^{1}$ Technische Universität Darmstadt, Schlossgartenstraße 8, 64289 Darmstadt, Germany \\ ${ }^{2}$ GSI Helmholtzzentrum für Schwerionenforschung GmbH, Planckstraße 1, 64291 Darmstadt, Germany
}

(Received 9 December 2013; published 3 December 2014)

\begin{abstract}
The electron cloud buildup and interaction with a train of relativistic, short proton bunches is studied using particle-in-cell codes. The simulation models describe the electron generation at the beam pipe wall as well as the wakefield behind the bunches. The study focuses on the space charge limited (saturated) cloud profile between the bunches and on the incoherent tune spread caused by the interaction of the saturated cloud with individual bunches. Analytical expressions describing the pinch of a saturated electron cloud are derived and compared to simulation results.
\end{abstract}

DOI: 10.1103/PhysRevSTAB.17.121001

PACS numbers: 29.27.Bd, 29.20.dk, 52.35.Qz

\section{INTRODUCTION}

Electron clouds limit the intensity of hadron beams in modern high energy colliders and synchrotrons (see e.g., [1]). In the CERN LHC, diverse effects related to electron clouds have been observed and studied at different bunch spacings [2]. The operation with $25 \mathrm{~ns}$ bunch trains is presently limited by strong electron cloud effects.

In the CERN super proton synchrotron and in the LHC, one attributes the observed slow transverse emittance growth as well as the rf phase shift of individual bunches in the train to the effect of the electron cloud $[3,4]$. Both effects depend again on the wakefield induced by a short, relativistic bunch in the electron cloud. The maximum electron cloud density is limited by its space charge electrical field (saturated electron cloud). The resulting density profile is far from being uniform. Moreover, it was shown [4] that the rf phase shift induced by a saturated electron cloud is smaller compared to an equivalent uniform electron cloud with the same total number of electrons. The effect of a saturated electron cloud profile on the transverse tune shift is the subject of the present study. In particular, we address the negative tune shifts caused by the strongly inhomogeneous electron cloud profile in an external dipole magnetic field. Assuming a uniform cloud density in elliptic geometry, the tune shift can be obtained analytically (see e.g., Ref. [5]). This assumption was used also in different studies (see for example Refs. [6-9]) to estimate the electron density based on the measured tune shifts. In Ref. [9] the authors reported a very good agreement between their simulation results and electron cloud densities estimated from the measured tune

petrov@ temf.tu-darmstadt.de

Published by the American Physical Society under the terms of the Creative Commons Attribution 3.0 License. Further distribution of this work must maintain attribution to the author $(s)$ and the published article's title, journal citation, and DOI. shifts. In the present study we will analyze the separate contributions from electron clouds in field-free and in dipole regions to the tune shift. In the field-free regions the electron cloud near the pipe center is usually rather uniform. On the contrary, in a dipole magnetic field the density profile becomes strongly nonuniform horizontally (see for example Refs. [10-13]). The density profile of the pinched electrons loses its radial symmetry as well. Moreover, after the scrubbing run in the LHC the electron cloud in the fieldfree regions is expected to vanish prior to the electron cloud in the dipole magnets [14]. Therefore accurate predictions of the (residual) cloud in the dipole magnets and its effect on the beam are of importance.

Most numerical studies of electron cloud effects are performed in two stages: buildup modeling and beam tracking. In buildup simulations the beam distribution is kept fixed and only the electron cloud production is modeled. This way the density profiles, the heat load [2] and the longitudinal and transverse wakefields can be obtained. As the electron cloud buildup and dynamics is much faster than the beam reaction, this decoupling is usually justified. Only in specific cases, like for example the buildup of an electron cloud from the residual gas in a coasting beam [15], a fully coupled buildup and beam dynamics model is required. For short LHC-type bunch trains the density profiles and wakefields obtained from the buildup simulations can be used in the beam tracking stage. In beam simulations often a homogeneous electron cloud profile is assumed and scans are performed over a range of initial cloud densities (see e.g., $[2,16])$. Such simulations are correct if the part of the cloud relevant for the interaction is approximately homogeneous. Still, one has to relate the obtained simulation result to the usually strongly inhomogeneous cloud profile in the pipe. We will address the differences between homogenous and realistic electron clouds.

In this study we focus on the incoherent tune spread caused by a saturated electron cloud. We investigate the 
difference between uniform (motivated by the theory) and realistic electron clouds for different bunch populations, with and without an external dipole magnetic field. Section II explicates the basic parameters of a saturated electron cloud. In Sec. III we examine analytically some features of the pinching of a ringlike electron cloud. Section IV provides the information about the numerical model. The results of buildup simulations are presented in Sec. V. The tune spreads induced by the cloud in field-free and in dipole magnets are investigated numerically in Sec. VI. Section VII concludes the paper.

\section{SPACE CHARGE LIMITED ELECTRON CLOUD}

The maximum density of an electron cloud is limited by its self-space-charge forces. During the buildup the electrons are accumulated until the cloud space charge potential exceeds the initial kinetic energy $W_{s}$ of the secondary electrons. New secondary electrons cannot penetrate into the central cloud region and remain in the vicinity of the pipe wall. The density of a homogeneous, saturated cloud can be easily obtained as (see Ref. [17])

$$
n_{\mathrm{sat}} \approx \frac{4 \varepsilon_{0} W_{s}}{e^{2} R_{p}^{2}},
$$

where $e$ is the electron charge, $R_{p}$ is the pipe radius, and $\varepsilon_{0}$ is the vacuum permittivity. For LHC parameters and $W_{s} \approx 2 \mathrm{eV}$ we obtain $n_{\text {sat }} \approx 10^{12} \mathrm{~m}^{-3}$. Equation (1) assumes a homogeneously distributed cloud. However, simulation results (Refs. $[4,13]$ ) show that a dense, thin $\left(d \ll R_{p}\right)$ electron layer develops near the wall. In a circular pipe between the bunches a ringlike electron cloud is formed near the pipe wall, together with a diluted homogeneous background density (see Fig. 2). Because, the longitudinal extent of the cloud (bunch spacing) is much larger than the transverse one (pipe diameter), the transverse fields are defined by the local cloud distribution in 2D. For such a ringlike electron cloud the average cloud density $n_{\text {sat }}$ could be much higher than Eq. (1). However, the layer thickness $d$ cannot be arbitrarily small because of the finite electron temperature. The realistic saturated cloud density must be obtained from numerical simulations. First, we will try to give some simple characteristics of the saturated cloud density in a circular pipe. The electric field at the pipe wall is $E_{p} \approx e n_{d} d / \varepsilon_{0}$, where $n_{d}$ is the density of the ringlike electron cloud and $d$ is the layer thickness. At the beam pipe,

$$
e E_{p} d=W_{s}
$$

determines the condition for the reflection of emitted electrons by the electron layer. Assuming that the layer is composed of low temperature $\left(k_{B} T_{e} \approx W_{s}\right)$, i.e., cold electrons we can equate the layer thickness to the Debye screening length $\lambda_{D} \approx d$, where

$$
\lambda_{D}^{2} \approx \frac{\varepsilon_{0} W_{s}}{n_{d} e^{2}} .
$$

For $W_{s} \approx 2 \mathrm{eV}$ and $n_{d} \gtrsim 10^{12} \mathrm{~m}^{-3}$ we obtain $\lambda_{D}<R_{p}$. The electrons with larger temperatures $\left(k_{B} T_{e} \gg W_{s}\right.$ or $\lambda_{D} \gg R_{p}$, where $k_{B}$ is the Boltzmann constant, $T_{e}$ is the electron temperature) form the diluted, homogeneous background.

\section{PINCHING OF A RINGLIKE ELECTRON CLOUD}

A rigid bunch of velocity $v_{0} \approx c$ interacts with a thin $\left(d \ll R_{p}\right)$ electron ring via its transverse electric field $E_{r}^{i}(R, z)$. Here we will ignore the beam's magnetic field as well as any magnetic field induced by the electrons. For a transverse Gaussian beam profile we obtain for the electric field

$$
E_{r}^{i}(r, z)=\frac{e \lambda(z, t)}{2 \pi \epsilon_{0} r}\left[1-\exp \left(-\frac{r^{2}}{2 \sigma_{r}^{2}}\right)\right],
$$

where $\lambda$ is the bunch line density, $r$ is the distance from the pipe center, and $\sigma_{r}$ is the rms bunch radius. We assume a round beam of radius $a=2 \sigma_{r}$. $a$ is the radius of the rms equivalent constant beam profile. The line density of the bunch is assumed to be Gaussian with

$$
\lambda(z)=\frac{N_{i}}{\sqrt{2 \pi} \sigma_{z}} \exp \left(-\frac{z^{2}}{2 \sigma_{z}^{2}}\right),
$$

where $N_{i}$ is the number of protons in the bunch, and $\sigma_{z}$ is the rms bunch length. The resulting electron-ring equation of motion is

$$
\frac{d^{2} r}{d z^{2}}+\kappa^{2}(r, z) r=0,
$$

where $\kappa(R, z)$ represents the focusing gradient due to the beam transverse electric field. Here we ignored the effect of electron space charge, assuming that the fast pinching of the electrons is dominated by the bunch transverse electric field. Furthermore we will assume that the bunch length $\sigma_{z}$ is short relative to the electron oscillation length in the bunch center $\kappa^{-1}(0)=\kappa_{0}^{-1}$ :

$$
\kappa_{0} \sigma_{z} \lesssim 1 \text {. }
$$

In this approximation the ring electrons at $R=R_{p}$ will simply receive a transverse momentum kick from the passing, short bunch [18]:

$$
R_{0}^{\prime}=-\frac{e}{m_{e} c^{2}} \int_{-\infty}^{\infty} E_{r}\left(R_{p}, s\right) d s=-\frac{2 r_{e} N_{i}}{R_{p}},
$$

where $r_{e}$ is the classical electron radius, $m_{e}$ is the electron mass. The resulting pinch motion for $z>0$ is governed by 


$$
R(z)=R_{p}-R_{0}^{\prime} z
$$

From this we can estimate the pinching point (distance behind the bunch center) as

$$
z_{p} \approx \frac{R_{p}}{R_{0}^{\prime}}=\frac{R_{p}^{2}}{2 r_{e} N_{i}}
$$

for LHC bunch parameters we always have $z_{p}>\sigma_{z}$ and the electron ring pinches well behind the bunch. The kinetic energy per electron in the ring is

$$
W_{\text {kin }}=\frac{m_{e} R_{0}^{\prime 2} c^{2}}{2} .
$$

In the presence of a strong external dipole field, electrons are confined to the magnetic field lines. The vertical kick seen by the electrons at the wall is $R_{0}^{\prime} \times \sqrt{1-x^{2} / R_{p}^{2}}$. All these electrons reach the horizontal plane $y=0$ at $z_{p}(x)=$ const given by Eq. (10).

The wake potential induced behind the bunch by the electron ring is

$$
\phi(z, r)=\frac{e \lambda_{e}}{2 \pi \varepsilon_{0}} \begin{cases}\ln \frac{R(z)}{R_{p}}, & 0 \leq r<R(z) \\ \ln \frac{r}{R_{p}}, & R(z) \leq r \leq R_{p},\end{cases}
$$

where $R(z)$ is the cloud radius at distance $z$ from the bunch peak, $\lambda_{e}$ is the electron cloud line density. The transverse electric field vanishes inside the electron ring,

$$
E_{r}^{e}(z, r)= \begin{cases}0, & 0 \leq r<R(z) \\ \frac{e \lambda_{e}}{2 \pi \varepsilon_{0} r}, & R(z) \leq r \leq R_{p} .\end{cases}
$$

For $\sigma_{z} \ll z_{p}$ there will be no transverse force on the bunch particles. Hence, we expect no or a strongly diminished effect of the pinched electron ring on the tune distribution, compared to an initially homogeneous cloud with the same total number of electrons.

It is important to note that in case of a strong external dipole magnetic field or a nonsymmetric pipe geometry this conclusion does not hold. The resulting asymmetric cloud density will generate a transverse force on the bunch particles, also without pinching.

In any case, the actual saturated electron cloud density and its interaction with the bunch should be obtained numerically. Our numerical model and the simulation results will be discussed in the next sections.

\section{NUMERICAL MODEL}

In order to study the effect of an electron cloud on a positively charged bunch, we utilize an electrostatic (ES) particle-in-cell (PIC) code with two-dimensional (2D) transverse beam-electron interaction. The simulations are typically performed in two steps: electron cloud buildup simulations and beam-particles tracking for a precalculated electron cloud distribution.

In the first step electrons are tracked in the plane perpendicular to the bunch direction of motion. The beam transverse density distribution on a 2D Cartesian grid is generated once in the beginning of each simulation. Subsequently, this distribution is scaled every time step according to the local beam line density. The electrons are represented by macroparticles. Their density is interpolated on the grid each time step and added to the beam density. The total charge density is then used to solve the 2D Poisson equation for the transverse potential. We have implemented a finite-difference cut-cell solver using the routines of the SuperLU library (Ref. [19]). During the simulation runs, electrons are pushed by the electric field and interact with the wall. A simplified version of the model given in Ref. [20] for the wall processes is used. True secondary electrons are produced at the wall with a $5 \mathrm{eV}$ Rayleigh distribution. The chosen energy parameter of the secondary electron energy distribution equals to the temperature in Ref. [21]. This distribution is simpler than the one given in Ref. [20] —it depends only on one parameter, but still gives zero emission probability at zero incident electron energy. The probability of true secondary emission on the energy is given by [20] $\delta_{t s}(x)=\delta_{t s, \max } s x /$ $\left(s-1+x^{s}\right)$, where $x=E / W_{S E Y \text {, max }}$ is the incident electron energy normalized to the position of maximum, $\delta_{t s, \max }$ is the maximum true secondary emission yield (SEY), $s$ is a fitting parameter. Rediffused electrons have uniform energy distribution ranging from zero to the energy of the incident electron. The dependence on the incident energy is $\delta_{\text {red }}(x)=\delta_{r d}\left(1-e^{-\left(E / E_{r}\right)^{r}}\right)$, where $\delta_{r d}$ is the rediffusion probability at infinity, $E_{r}$ and $r$ are the fit parameters. An analytical formula for the reflection dependence on the energy is taken from Ref. [22]: $\delta_{e l}(E)=$ $\left(\sqrt{E}-\sqrt{E+E_{0}}\right)^{2} /\left(\sqrt{E}+\sqrt{E+E_{0}}\right)^{2}$, where $E_{0}=150 \mathrm{eV}$. Following Ref. [22] the reflection probability at zero energy is set to $\delta_{r e}(0)=1$. In our studies we neglect the SEY dependence on the angle of incidence. The angular distribution of true secondary and rediffused electrons follows a cosine. The weight of newly produced electrons is equal to the weight of an incident electron. In our simulations a maximum number of macroelectrons $N_{\max }$ is set. If this number is exceeded, then part of the macroelectrons are deleted and the weight is proportionally increased. When a macroelectron hits the wall the probabilities of all the processes are calculated according to the previously mentioned formulas. Afterwards, if $\delta_{t s}(E)>1$, then an integer number of true secondaries floor $\left[\delta_{t s}(E)\right]$ is generated. Hereafter, a random number $g$ with the uniform distribution from 0 to 1 is generated. If $g$ is less than the fractional part of $\delta_{t s}(E)$, then an additional electron is produced. Additionally, two random numbers with the same uniform distribution are generated to produce reflected and rediffused electrons. We assume that all these 
processes can be treated independently. During the buildup the electron cloud density $\rho_{e, 2 D}(x, y, t=z / c)$, the potential $\phi_{e, 2 D}(x, y, t=z / c)$ and the cloud transverse electric field $E_{e, 2 D}(x, y, t=z / c)$ are saved for each beam slice and can be used in the beam tracking. In all the simulations the buildup is initiated from a low-density homogeneous electron cloud. No primary electrons are produced in the buildup simulation.

In particle tracking simulations the bunch ions are represented by macroparticles distributed along the bunch slices on a grid according to the local beam density $\rho_{i}(x, y, z)$. The interaction of the bunch with the electron cloud is studied separately in field-free and in dipole regions. In each particular simulation the bunch is affected either by an electron cloud in the field-free section or by a cloud in the dipole region but not by both together. Since we focus on the incoherent beam particle tune, the tracking is performed for a small number of turns. Moreover, we track only one bunch under the influence of the saturated cloud. For a stable bunch the density and the 3D profile of the electron cloud seen by the bunch will be the same from turn to turn. For this reason, instead of using a macroparticle electron cloud we use the 3D density profile and the corresponding electric field obtained from the buildup simulations. The transverse field of an electron cloudsaved for each bunch slice in the first simulation step-is used to evolve the beam particles.

Afterwards, a simple transfer matrix with constant tunes is used to transport the beam particles to a new electron cloud location. Thereby we assume that the electron cloud is the only source of tune spreads. The scheme for the tune footprint calculation used in our simulation can be found in Ref. [23]. We neglect the synchrotron motion as well as the coupling between the motion in $x$ and $y$ directions. Thus, in each plane a simple $2 \times 2$ matrix is employed. The particle coordinates are then given by

$$
\begin{aligned}
\left(\begin{array}{c}
x_{n+1} \\
x_{n+1}^{\prime}
\end{array}\right)= & \left(\begin{array}{cc}
\cos (\mu) & \frac{L}{2 \pi Q_{x}} \sin (\mu) \\
-\frac{2 \pi Q_{x}}{L} \sin (\mu) & \cos (\mu)
\end{array}\right) \\
& \times\left[\left(\begin{array}{c}
x_{n} \\
x_{n}^{\prime}
\end{array}\right)+\left(\begin{array}{c}
0 \\
\Delta x_{e}^{\prime}
\end{array}\right)\right],
\end{aligned}
$$

where $x$ is the transverse coordinate, $x^{\prime}$ is the transverse momentum, $\Delta x_{e}^{\prime}$ is the momentum kick, $n$ denotes the turn number, $Q_{x}$ is the betatron tune, and $\mu$ is the phase advance between the eclouds. Following Ref. [23] one can reconstruct the transfer matrix $M$, including the electron cloud kick, from the stored particle coordinates,

$$
M=\left(\begin{array}{cc}
\cos \left(\mu_{\text {new }}\right)+\alpha \sin \left(\mu_{\text {new }}\right) & \beta \sin \left(\mu_{\text {new }}\right) \\
-\gamma \sin \left(\mu_{\text {new }}\right) & \cos \left(\mu_{\text {new }}\right)-\alpha \sin \left(\mu_{\text {new }}\right)
\end{array}\right),
$$

where $\beta, \gamma, \alpha$ are the Twiss parameters at the position of the cloud and $\mu_{\text {new }}=$ arccos is the modified phase advance for an individual particle. The resulting tune shift of the particle is

$$
\Delta Q_{x}=\frac{\mu_{\text {new }}-\mu}{2 \pi} .
$$

Typically, we use only one interaction point. The kick due to an electron cloud is calculated as follows:

$$
\Delta x_{e}^{\prime}=\frac{e E_{x}^{e}(x, y) L}{\gamma_{0} m_{p} c^{2}}
$$

where $L$ is the length of the electron-cloud containing region, $\gamma_{0}$ is the Lorentz factor, and $m_{p}$ is the proton mass.

\section{SIMULATIONS OF THE ELECTRON CLOUD BUILDUP AND SATURATION}

The relevant simulation parameters for LHC-type bunches and parameters are listed in Table I. Electron cloud simulation studies for LHC focusing on different aspects were presented in Refs. [1-4,21,24], for example. In our study we chose beam and pipe parameters close to the LHC case in order to provide results for the electron densities and the tune shifts which are close to the expected values in an existing machine. However, detailed and quantitatively accurate electron cloud predictions for specific machine sectors depend on the detailed geometry of the pipe. Geometries with different horizontal and vertical dimensions yield different field gradients in $x$ and $y$ directions even for uniform unperturbed electron clouds. In the Appendix we compare results obtained for the actual LHC pipe geometric with the circular pipe used in our study. It is shown that, although the exact values differ, the results for the tune shifts agree qualitatively. In our study

TABLE I. Simulation parameters for LHC-type bunches.

\begin{tabular}{lc}
\hline \hline Bunch length, $\sigma_{z}[\mathrm{~m}]$ & 0.1 \\
Bunch radius, $\sigma_{r}[\mathrm{~m}]$ & $10^{-3}$ \\
Bunch population, $N_{i}$ & $2.5 \times 10^{10}-6 \times 10^{11}$ \\
Bunch spacing [ns] & 25 \\
Pipe radius, $R_{p}[\mathrm{~m}]$ & $2 \times 10^{-2}$ \\
Magnetic field, B [T] & 0.1 \\
Maximum total SEY, $\delta_{\max }$ & $1.4,2.1$ \\
Energy of $\delta_{\max }, W_{\mathrm{SEY}, \max }[\mathrm{eV}]$ & 250 \\
$s$ for true secondaries & 1.8 \\
Reflection probability & 1.0 \\
$E_{0}$ for reflected electrons & 150 \\
Rediffusion probability, $\delta_{r d}$ & 0.7 \\
$E_{r}$ for rediffused electrons $[\mathrm{eV}]$ & 5 \\
$r$ for rediffused electrons & 1 \\
Ring circumference, C $[\mathrm{m}]$ & $2.7 \times 10^{4}$ \\
Fraction of the ring with EC & 0.1 \\
Betatron tunes $Q_{x} / Q_{y}$ & $65.32 / 63.27$ \\
Lorentz factor, $\gamma_{0}$ & 450 \\
\hline \hline
\end{tabular}


TABLE II. Computational parameters.

Maximum number of macroelectrons

Longitudinal slice length, $d z[\mathrm{~m}]$

Grid dimensions

$2 \times 10^{5}$

$8 \times 10^{-4}-2 \times 10^{-3}$

$150 \times 150$

any asymmetry of the incoherent tune shift and spread arises entirely from the evolution of the electron cloud distribution itself and not from the pipe geometry. This facilitates the interpretation of our results. Concerning the production mechanisms at the pipe wall we decided to include rediffused electrons because Ref. [25] indicates the contribution of rediffused electrons even after scrubbing remains unchanged. The chosen fitting parameters are close to the ones given for stainless steel in Ref. [20]. Our intent is not to simulate the particular setup in all details, but to study sufficiently realistic density profiles that resemble those in a real high-energy hadron machine. The computational parameters are given in Table II. For all the presented simulations only one interaction point was assumed. Our convergence studies indicated that in cases of 1 and 100 interaction points the difference in tune shifts is of the order of $5 \%$.

A typical evolution of the electron line density along the bunch train is shown in Fig. 1. The electron cloud buildup starts out exponentially and saturates due to space charge. The simulation results for a circular beam pipe and LHCtype bunch parameters are presented in Fig. 2. Shown is the electron density snapshot of the saturated cloud $6 \times \sigma_{z}$ before the bunch. One can clearly identify the dense electron sheath at the pipe wall and the diluted cloud density ( 1 order of magnitude) around the pipe center. The corresponding temperature profile is shown in Fig. 3. At the pipe wall the temperature drops to values close to $W_{s}$ (which is chosen as $5 \mathrm{eV}$ in the simulations). At the center the cloud is much hotter $(>10 \mathrm{eV})$. In the energy spectrum

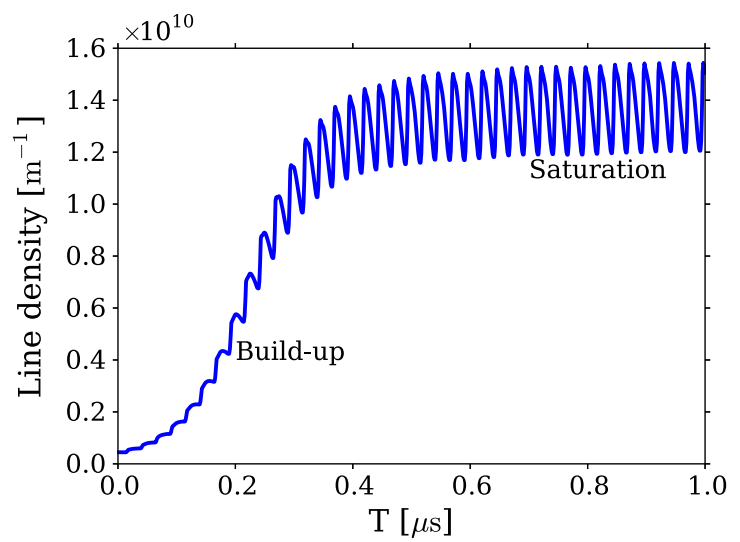

FIG. 1. Electron cloud buildup in the presence of an external dipole magnetic field. One can see the initial exponential phase and the saturation. The latter is studied in this paper. $N_{i}=10^{11}$ and $\delta_{\max }=1.4$.

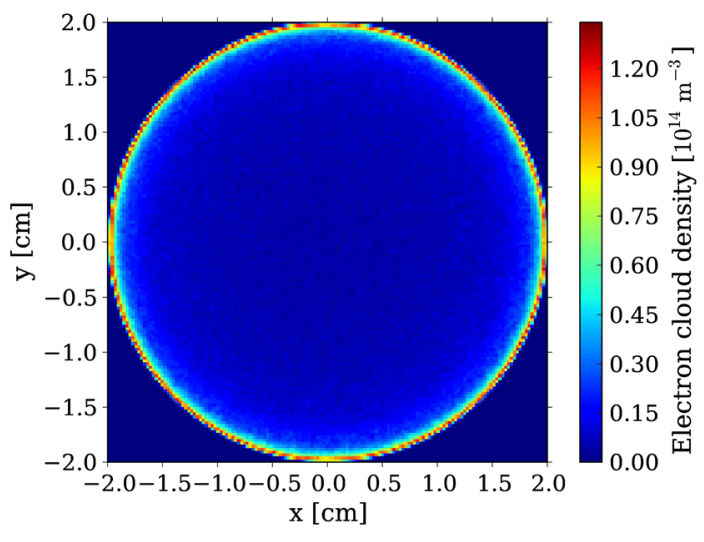

FIG. 2. Snapshot of the electron density of the saturated cloud $6 \times \sigma_{z}$ before the bunch peak (25 ns spacing) in a circular pipe.

(not shown) one can clearly distinguish the cold and hot electron components.

The saturated electron line density in the pipe cannot be described easily in terms of Eq. (1), which does not include dependencies on the SEY or on the bunch population. Figure 4 shows the line density of the saturated cloud (blue curve) together with the heat load as a function of the bunch population. One can see that the line density reaches its maximum around $N_{i}=1 \times 10^{11}$ and drops afterwards. This is very close to the bunch population of $N_{i}=1.1 \times 10^{11}$ at which sheath electrons gain exactly $E_{\mathrm{SEY}, \max }$ [according to Eq. (11)]. A minimum is reached at $N_{i}=4 \times 10^{11}$ and a slow density growth is observed at higher intensities. These results agree qualitatively with Ref. [26]. The heat load shown in the figure was calculated using the energy deposited on the wall during the last $25 \mathrm{~ns}$. Contrary to density, the heat load stays almost constant for $N_{i}>2 \times 10^{11}$. This density behavior can be explained (see Ref. [26]) if we consider the energy gained by the electrons depending on their impact factor (the initial distance to the beam axis). For low beam intensities the energy gain of the

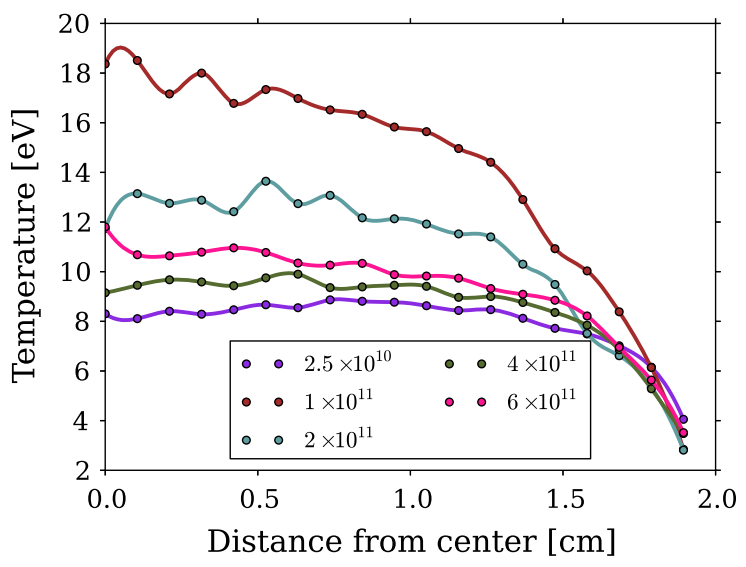

FIG. 3. The electron temperature profile of the saturated cloud $6 \sigma_{z}$ before the bunch (25 ns spacing) in a circular pipe for different bunch populations. 


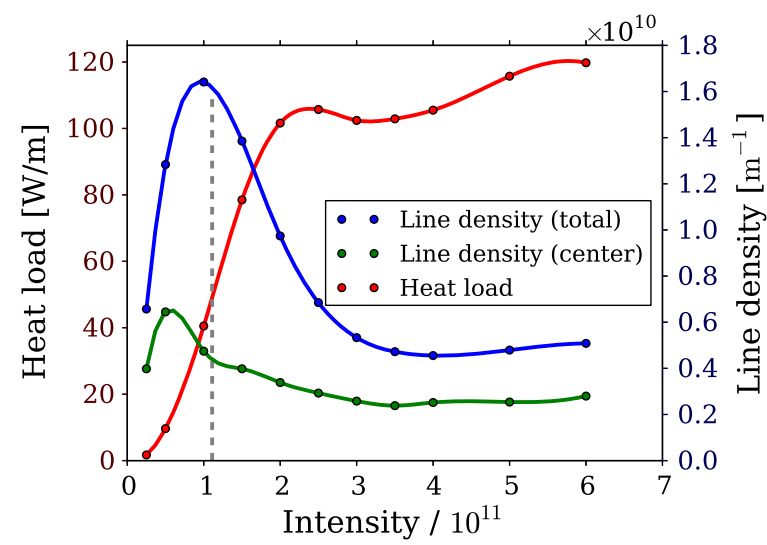

FIG. 4. The saturated electron cloud line density (blue) and heat load (red) against the intensity in the absence of magnetic fields. The dashed grey line shows the intensity at which the energy gain of the sheath electrons is $W_{\mathrm{SEY}, \max }$ [Eq. (11)]. The SEY is chosen as $\delta_{\max }=1.4$. The green curve represents the line density corresponding to the diluted part of the cloud.

electrons is smaller than the energy maximum of the SEY $\left(W_{\text {SEY,max }}\right)$. With increasing bunch population a group of electrons with exactly $W_{\mathrm{SEY} \text { max }}$ arises. The impact factor $b$ for this group increases with intensity and finally reaches the pipe radius $R_{p}$. Because the density of the electron cloud has its maximum near the wall, the generation of secondary electron reaches its maximum. If the intensity increases further, the sheath electrons at the wall gain an energy which is larger than $E_{\mathrm{SEY} \text {,max }}$ and the production of secondaries decreases. Figure 4 also shows the diluted line density of the saturated cloud (green curve) as a function of the bunch population. The diluted cloud density is obtained from the area inside the radius $2 \times \sigma_{r}$. One can see that the density of the diluted cloud has a maximum shifted relative to the average density.

In the case of a strong dipole magnetic field, the maximum in the line density is less defined (see Fig. 5), mainly because of the lower energy gain of the electrons in the electric field of the bunch. It should also be noted that for lower intensities the line density of the diluted cloud, as defined previously, is actually larger than the total line density. This is because the cloud is localized in a thin stripe as shown in Fig. 6. The pinch profiles in the horizontal plane are shown in Fig. 7. The pinching points given by Eq. (10) are also depicted in this figure. One can see that the electron cloud density peaks along $x$ for $z_{p}$ predicted by Eq. (10). These are the electrons from the wall reaching $y=0$.

As in the field-free sector, the dense and cold electron layer can also be identified in a dipole sector. One can see that the density profiles differ significantly. They depend on the details of the electron energy spectra and the space charge distribution in the pipe. At low intensities the cloud is localized within a single stripe in the central region of the pipe. With increasing intensity the stripe splits into two.

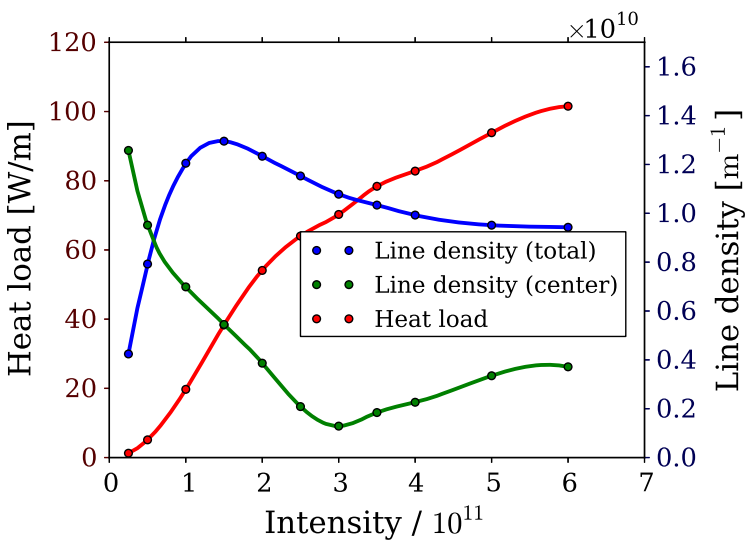

FIG. 5. The saturated electron cloud line density (blue) and heat load (red) against the bunch population in the presence of external dipole magnetic field. The SEY is chosen as $\delta_{\max }=1.4$. The green curve represents the line density corresponding to the diluted part of the cloud.

Similar stripe structure of the electron cloud profile has been described in Refs. [10,12,13]. At intensities well beyond the ones presently achieved in the LHC the cloud is distributed across the whole pipe diameter.

The densities obtained in the simulations are higher than the ones presented in Refs. [26,27]. This is due to the chosen higher elastic-reflection probability and the rediffusion. One also has to take into account that we assume a simplified geometry, an exact agreement cannot be expected. To justify that our model gives realistic results we compared the heat load with already published data. In Ref. [21] the heat load for similar conditions and for $25 \mathrm{~ns}$ bunch spacing was simulated. For a bunch population of $10^{11}$ the obtained heat load was approximately 0.8 and $2 \mathrm{~W} / \mathrm{m}$ for the dipole and field-free sections, respectively. In Ref. [21] the heat load was calculated averaged over the LHC circumference for two trains with 72 bunches separated by eight empty buckets. In Ref. [2] the average measured heat load in the LHC for $25 \mathrm{~ns}$ bunch trains was approximately $0.7 \mathrm{~W} / \mathrm{m}$. To compare the values discussed above with our results, one needs to multiply our heat load

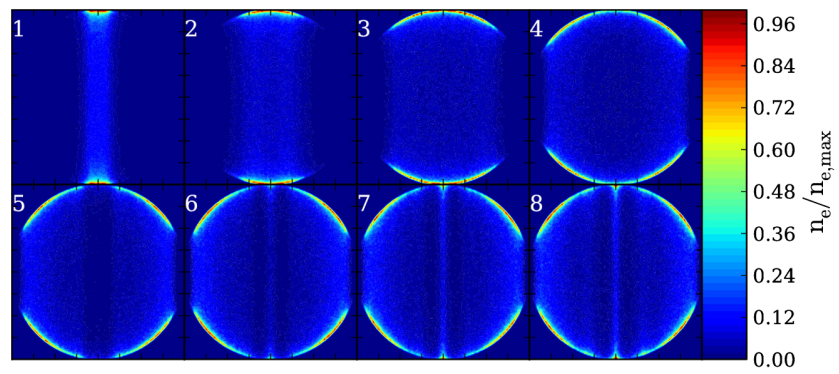

FIG. 6. Snapshot of electron cloud profiles $6 \times \sigma_{z}$ before the bunch peak in the presence of external dipole magnetic field for $2.5 \times 10^{10}, 5 \times 10^{10}, 1 \times 10^{11}, 2 \times 10^{11}, 3 \times 10^{11}, 4 \times 10^{11}$, $5 \times 10^{11}$, and $6 \times 10^{11}$ bunch populations in round LHC like pipe. $\delta_{\max }=1.4$. 


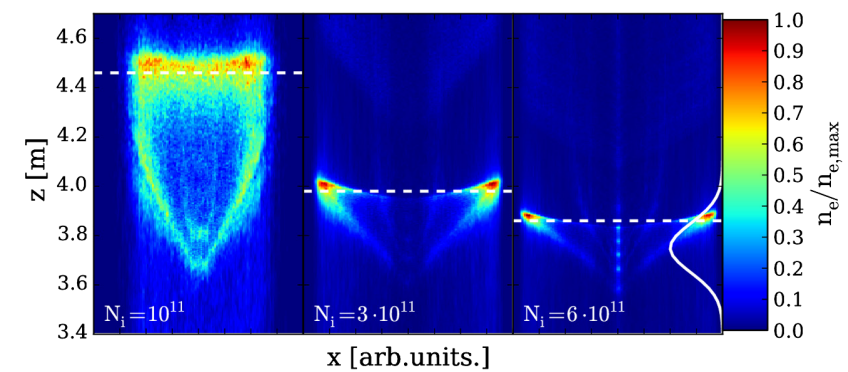

FIG. 7. Electron cloud pinching in horizontal plane $y=0$ crossing the pipe center (external magnetic field is on). From left to right: $N_{i}=10^{11}, N_{i}=3 \times 10^{11}$ and $N_{i}=6 \times 10^{11}$. The solid gray line shows the position of the bunch and its profile. The dashed lines depict the pinching points given by Eq. (10). Direction of motion is downwards.

by the full length of the given trains and divide by the LHC circumference. In this case our simulations give approximately $0.8 \mathrm{~W} / \mathrm{m}$ for the dipole and $1.6 \mathrm{~W} / \mathrm{m}$ for the fieldfree sections. In the Appendix a discussion of a realistic LHC pipe geometry case is presented.

\section{INCOHERENT TUNE SPREAD DISTRIBUTION INDUCED BY A SPACE CHARGE LIMITED CLOUD}

The electron cloud causes an incoherent tune spread of the particles in the bunch (Ref. [2]). The tune spread depends on the density profile of the electron cloud at the position of the bunch. In this section we present simulation results for the incoherent tune spread in LHC-type bunches. As it was shown in the previous section the transverse cloud profile is highly nonuniform with a dense layer near the wall. This dense sheath reaches the pipe center later than the electrons in the diluted part of the cloud. We will analyze the contribution of the electrons that do not reach the beam core.

Therefore we will compare the tune spread induced by a "real" electron cloud obtained from our buildup simulations (nonuniform cloud) with the tune shift obtained from an initially uniform cloud.

First, we compare the tune footprints obtained for nonuniform and for the equivalent uniform electron clouds. The uniform cloud density is equal to the average density of the nonuniform cloud (the line density for both clouds is equal). In Figs. 8 and 9 it is seen that for LHC-type bunches the tune spreads induced by the uniform cloud are larger than due to the nonuniform cloud profile. This is because the ringlike dense electron sheath pinches well behind the bunch.

Second, we compare the nonuniform cloud to a uniform cloud density corresponding to the diluted, central part of the nonuniform cloud (diluted cloud). The tune footprints induced by the initially diluted cloud are shown in Figs. 10 and 11 . One can see that in a field-free section the diluted

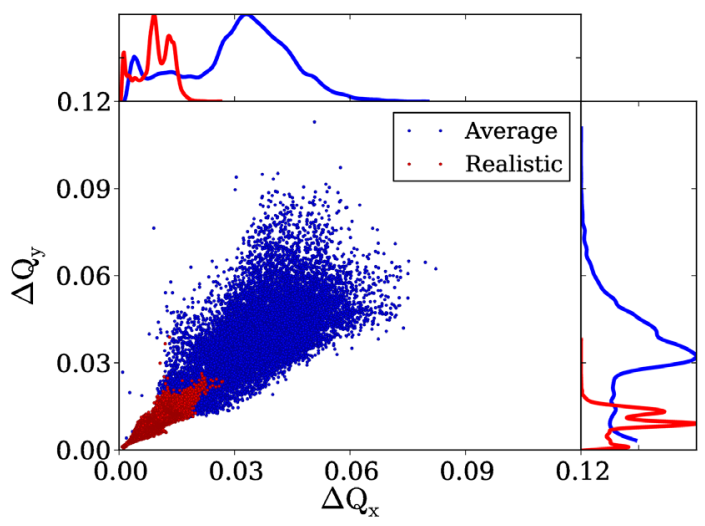

FIG. 8. Tune spreads for the LHC-type bunch in round geometry without magnetic field. Uniform cloud density is equal to the average realistic cloud density. Bunch population $N_{i}=10^{11}, \delta_{\max }=2.1$.

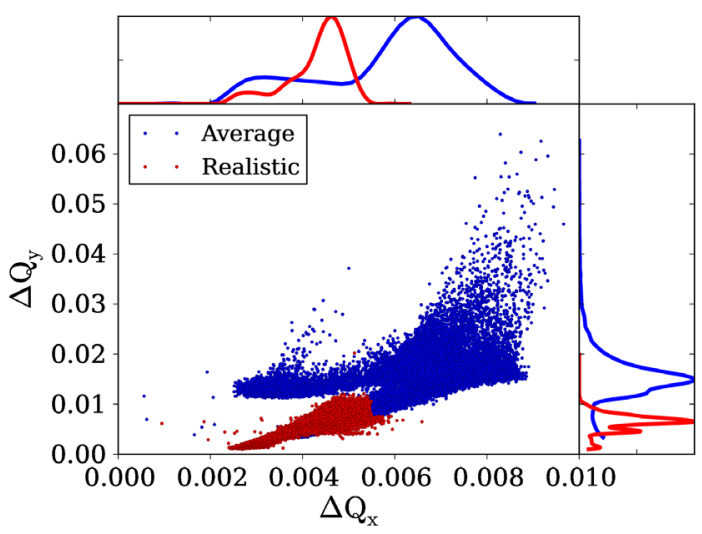

FIG. 9. Tune spreads for the LHC-type bunch in round geometry with magnetic field $B=0.1 T$. Uniform cloud density is equal to the average realistic cloud density. Bunch population $N_{i}=10^{11}, \delta_{\max }=2.1$.

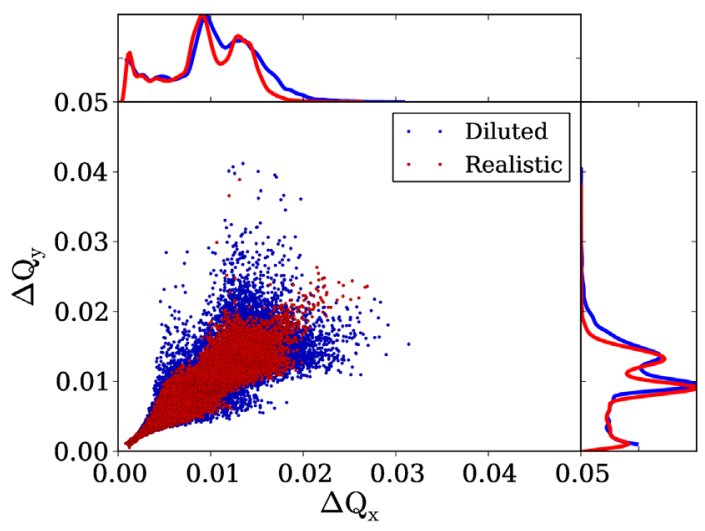

FIG. 10. Tune spreads for LHC-type bunch in a round pipe geometry without magnetic field. The blue dots represent the tune distribution induced by the diluted cloud. The red dots correspond to the tune distribution induced by the nonuniform cloud. The bunch population is $N_{i}=10^{11}$ and the SEY $\delta_{\max }=2.1$. 


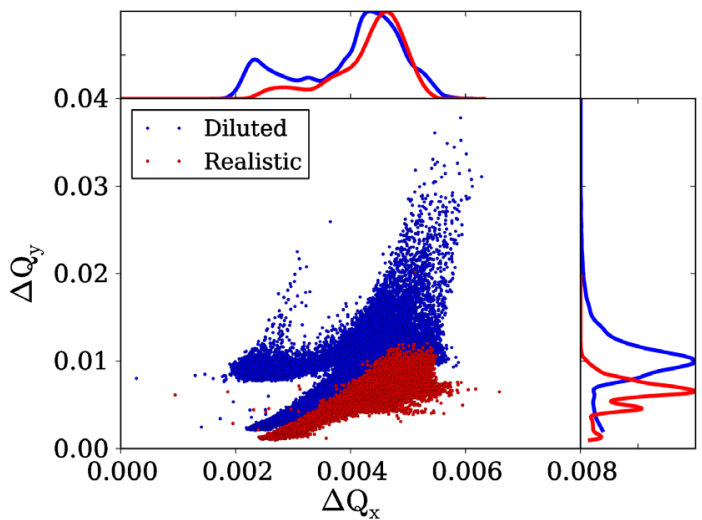

FIG. 11. Tune spreads for LHC-type bunch in a round pipe geometry with magnetic field. The blue dots represent the tune distribution induced by the diluted cloud. The red dots correspond to the tune distribution induced by the nonuniform cloud. The bunch population is $N_{i}=10^{11}$ and the SEY $\delta_{\max }=2.1$.

cloud leads to tune spreads similar to the nonuniform cloud. However, in a dipole region the resulting tune diagrams deviate significantly, especially in the vertical plane.

We have chosen the models above being motivated by the analytical formula for the electron-cloud induced tune shift [5]:

$$
\Delta Q_{x}(z)=\frac{r_{p} L \bar{\beta}_{x} b \bar{d}}{\gamma_{0}(a+b)} \rho(z)
$$

where $L$ is the length of the electron-cloud containing region, $\bar{\beta}_{x}$ is the average beta function, $a$ and $b$ are the transverse pipe dimensions, $r_{p}$ is the proton classical radius, $\bar{d}$ is the average electron density and $\rho(z)$ is the normalized electron density profile along the bunch. From the above formula the electron cloud density averaged over the bunch results as

$$
\bar{d} \bar{\rho}=\frac{\gamma_{0}}{r_{p} L}\left(\frac{\Delta Q_{x}}{\bar{\beta}_{x}}+\frac{\Delta Q_{y}}{\bar{\beta}_{y}}\right) .
$$

As it was mentioned in the Introduction, the above tune shift formula, in one form or another, is used and cited in a number of publications [6-9]. Attempts to study the pinch effect on the tune shift analytically were performed in Ref. [28]. All the analytical approaches have in common that the tune shifts in both directions are proportional to the electron density. Our uniform and diluted cloud models are closely related to the analytic approaches. However, as shown in Figs. 4 and 5 the question about the correct density to be used in Eq. (18) remains. The densities obtained from the average and the diluted cloud models scale differently with the bunch population, especially in a dipole region.

\section{A. Intensity dependence of the tune shift and spread}

In the following we will analyze the rms tune spread as a function of the bunch population. Figure 12 depicts the tune shift and its rms deviation as a function of intensity for nonuniform and uniform clouds in a round pipe geometry. One can see that the tune shifts and spreads for the nonuniform cloud and for the diluted uniform cloud are similar up to $N_{i} \approx 1 \times 10^{11}$. For larger intensities the diluted cloud underestimates the tune shift and spread. There is no well-defined threshold because the cloud density grows smoothly with distance from the center (Fig. 2). The uniform average cloud (same line density) strongly overestimates the tune shift and spread for LHC intensities. The curves for the tune shift and spread in Fig. 12 can be compared with the line densities shown in Fig. 4. There is a clear correlation between the tune shifts and the line densities for the diluted cloud. One can see an increasing discrepancy between the realistic and diluted electron cloud models for $N_{i}>10^{11}$. This is because additional electrons from the outside denser regions of the realistic cloud are getting involved into the pinching process.

Figures 13 and 14 show the tune shifts and spreads in the horizontal and the vertical direction in the presence of a strong external dipole magnetic field. Again a round pipe geometry is chosen. In the horizontal direction the results obtained for the nonuniform cloud (blue curves) agree rather well with the ones for the diluted cloud (green curves) up to $N=2 \times 10^{11}$. Moreover, the horizontal tune shift for the nonuniform cloud reaches a negative value about $N \approx 3 \times 10^{11}$.

The negative tune shift arises because of the larger electron cloud density to the left and to the right from the beam center (Fig. 6). The (uniform) diluted part of the

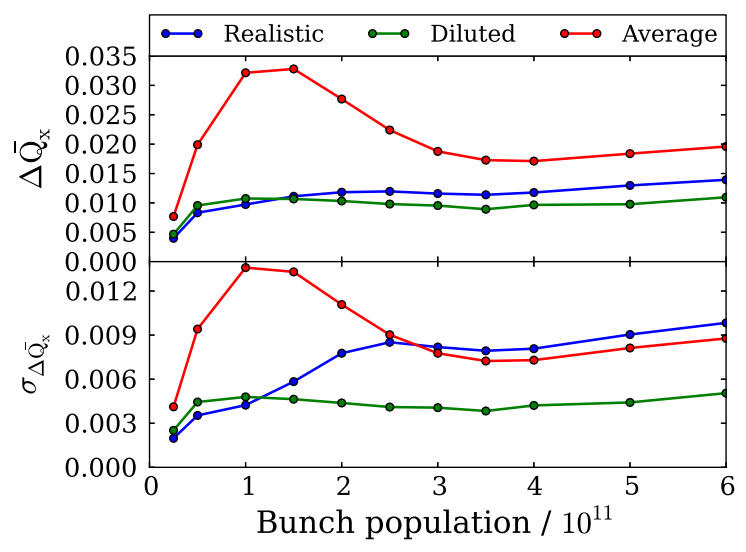

FIG. 12. The tune shift (upper graph) and its standard deviation (lower graph) as a function of bunch population in a field-free region. The blue curve shows the results for a nonuniform cloud. The green curve shows the results for the uniform, diluted cloud. The red curve corresponds to the uniform cloud. The SEY is $\delta_{\text {max }}=1.4$. 


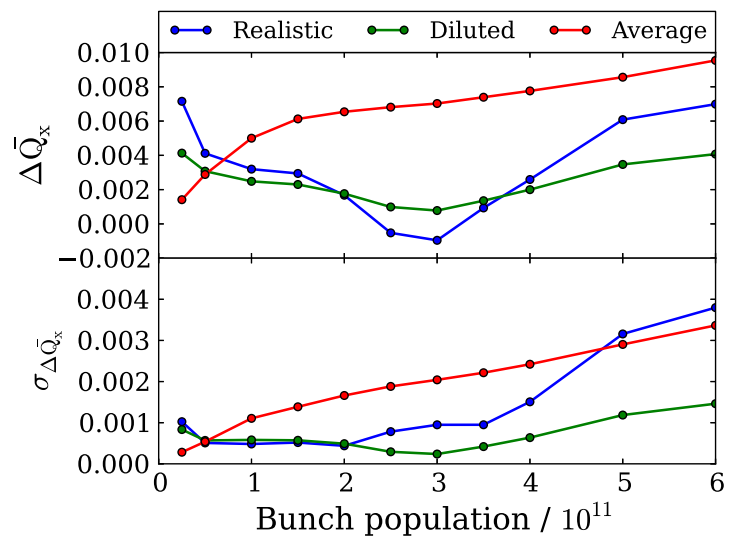

FIG. 13. The horizontal tune shift (upper graph) and its standard deviation (lower graph) as a function of bunch population in a dipole magnet. The blue curve shows the results for a nonuniform cloud. The green curve shows the results for the uniform, diluted cloud. The red curve corresponds to the uniform cloud. The SEY is $\delta_{\max }=1.4$.

cloud always causes a positive tune shift. The contribution of the nonuniform cloud, outside of the beam center, will always lead to a negative tune shift in one direction and a positive one in the other direction. This is simply a consequence of $\partial E_{x} / \partial x+\partial E_{y} / \partial y=0$ for the electric fields in two dimensions. In the general case the passing bunch sees a superposition of the fields from the central part of the electron cloud and from the nonuniform outside parts. If the nonuniform parts dominate the tune shift can be negative in one direction.

In the radially symmetric case, the local electron density at the bunch position defines the electric field and the corresponding tune shift. The dense sheath does not contribute to the tune shift, unless it reaches the pipe center at the position of the bunch. Usually the radial symmetry is broken, either due to a external magnetic field or due to an asymmetric pipe geometry. Thus, the contribution of the dense sheath may become more important for the tune calculations. Except for round field-free pipes, at high intensities one should always account for the realistic electron distribution from the buildup simulations together with the proper boundary conditions.

Figure 15 shows the reconstructed electron cloud density (averaged over the bunch) in the dipole region. To obtain this figure the results shown in Figs. 13 and 14 were plugged into Eq. (19). One can see that the curves for the diluted and realistic models yield nearly the same electron cloud density up to $N \approx 3 \times 10^{11}$. Afterwards, for higher bunch populations, there is an increasing discrepancy between the two models. A possible reason is the increase of the electron cloud density towards the wall-more electrons from this denser part contribute to the pinch for higher bunch population. In case of the realistic cloud model the actual pinched electron density, averaged over the bunch, agrees very well with the density reconstructed

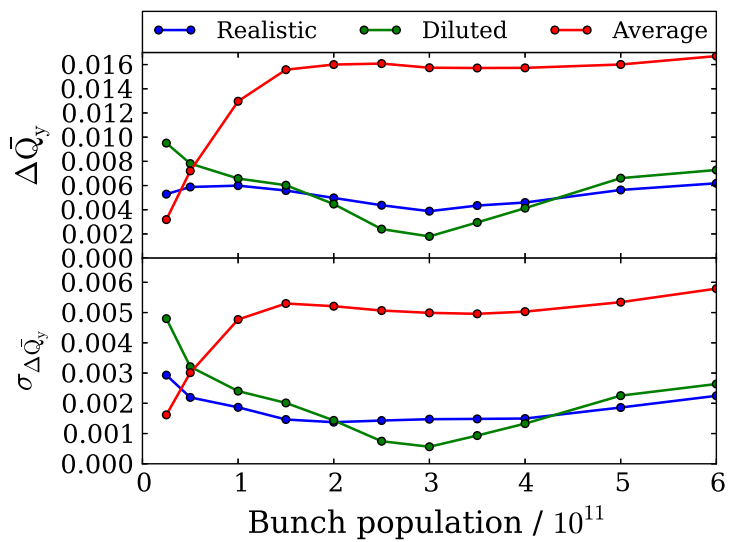

FIG. 14. The vertical tune shift (upper graph) and its standard deviation (lower graph) as a function of bunch population in a dipole magnet. The blue curve shows the results for a nonuniform cloud. The green curve shows the results for the uniform, diluted cloud. The red curve corresponds to the uniform cloud. The SEY is $\delta_{\max }=1.4$.

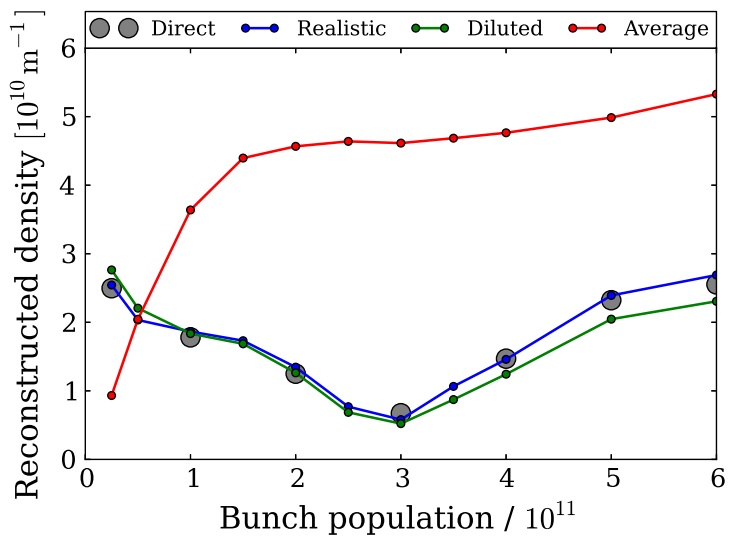

FIG. 15. Electron density in a dipole region reconstructed from the tune shifts using Eq. (19). The electron cloud density calculated directly from the macroparticle distribution in the buildup simulations is shown in grey.

from the tune shifts. Those results obtained for the chosen example parameters are also consistent with the findings in Ref. [9], where an impressive agreement between the electron cloud density reconstructed from the measured tune shifts and detailed buildup simulations has been reported. Our results indicate that for lower bunch populations or nonsaturated electron cloud densities detailed buildup simulations might not be required. Also if the electron cloud is predominately in field-free sections, the diluted cloud model could be a good approximation.

\section{SUMMARY AND CONCLUSION}

We have investigated the incoherent tune spread induced by an electron cloud in relativistic proton bunches in a circular geometry by means of a 2D electrostatic simulation 
model. For this purpose electron cloud buildup simulations were performed under conditions similar to the LHC. We found that for a broad beam intensity range (also covering the LHC parameters) the saturated electron cloud between bunches forms a dense, cold sheath near the pipe wall (nonuniform cloud). This holds for a field-free pipe as well as for a pipe placed in a dipole magnet.

The observed dependence of the average electron cloud density on the bunch population is similar to Ref. [26]. However, the central density seen by the head of the bunch has a nontrivial connection with the average density and the beam intensity. As an example, for intensities above the nominal LHC bunch population the central cloud density in the dipole region reaches a minimum (see Fig. 5).

We analyzed the characteristic dense electron sheath at the pipe wall and presented simple analytical estimates for its thickness and its pinching distance for a circular geometry. Our simulation results agree rather well with those estimates for LHC intensities and larger. Under LHC conditions the electrons at the wall pinch well behind the bunch.

The tune spreads induced by a pinching, initially nonuniform cloud were compared to initially uniform clouds. The uniform cloud parameters were chosen to resemble the ones used in the analytical theory. We found that the model of an average uniform cloud overestimates the tune spreads and tune shifts in almost every case. In a radially symmetric field-free section a diluted, uniform cloud density provides a better approximation of the results obtained for the real nonuniform cloud. In an external dipole magnetic field and for sufficiently high bunch populations the tune shift and spread cannot be reproduced by uniform cloud models.

For sufficient bunch populations the electron cloud in a dipole region can form stripes with negligible density at the pipe center. The stripe structure of the electron cloud in the presence of an external magnetic field is already a well-known phenomenon (described for example in Refs. [10-13]). However, it is important to note that the strongly inhomogeneous electron density profile of the pinching stripes results in a strongly asymmetric tune shifts, which can even be opposite in sign. In such a case the tune shift in the horizontal or in the vertical directions separately cannot be used to estimate the electron cloud density.

However, we find that when the sum of the horizontal and vertical tune shifts is used to calculate the central electron cloud density, then the diluted cloud model and realistic simulation give similar reconstructed densities. More in general and in particular in dipole regions, one should always perform detailed buildup simulations and include features of the electron cloud profile outside of the beam in the calculation of the tune shifts. Based on our present and previous (Refs. [4,13]) studies we conclude that beam simulation of incoherent or coherent electron cloud effects should utilize, whenever possible, realistic cloud distributions obtained from detailed electron cloud buildup models. For certain parameter regimes, as for example in field-free sections or for lower bunch populations, detailed buildup simulations might not be required and uniform cloud models, as presented in this contribution, could be a good approximation.

\section{ACKNOWLEDGMENTS}

The work has been supported by the Federal Ministry of Education and Research (Germany) under Contract No. $05 \mathrm{H} 12 \mathrm{RD} 7$. We would like to thank the referees for the proposed improvements.

\section{APPENDIX: SIMULATIONS FOR THE LHC PIPE GEOMETRY}

The aim of this section is to show that for a realistic LHC pipe geometry [29] (half-width $R_{x}=22 \mathrm{~mm}$ and halfheight $h_{y}=18 \mathrm{~mm}$ ), the saturated electron cloud has similar features as in a circular pipe. Other simulation parameters are listed in Tables I and II. Figures 16 and 17 show electron-cloud density snapshots in field-free and dipole-magnet regions. One can see the formation of a

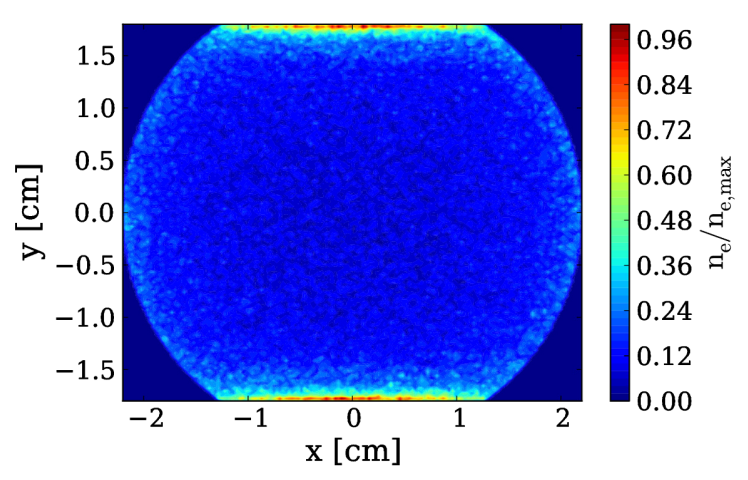

FIG. 16. Snapshot of electron cloud density $6 \times \sigma_{z}$ before the bunch in a field-free region. The LHC pipe geometry is used. Bunch population is $10^{11}$.

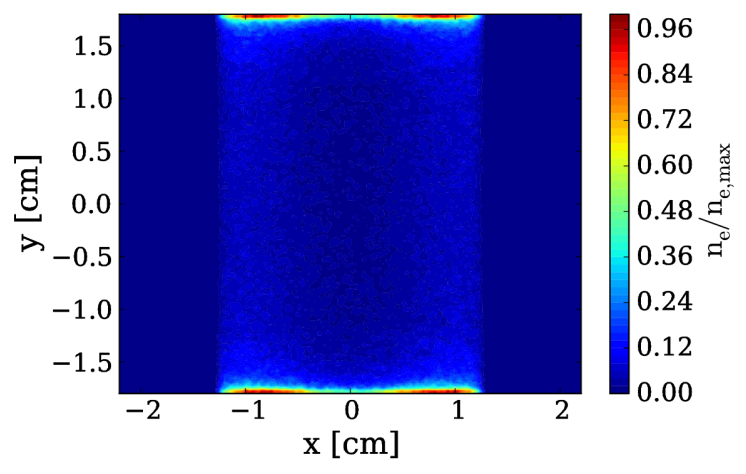

FIG. 17. Snapshot of electron cloud density $6 \times \sigma_{z}$ before the bunch in the presence of an external magnetic field. The LHC pipe geometry is used. Bunch population is $10^{11}$. 


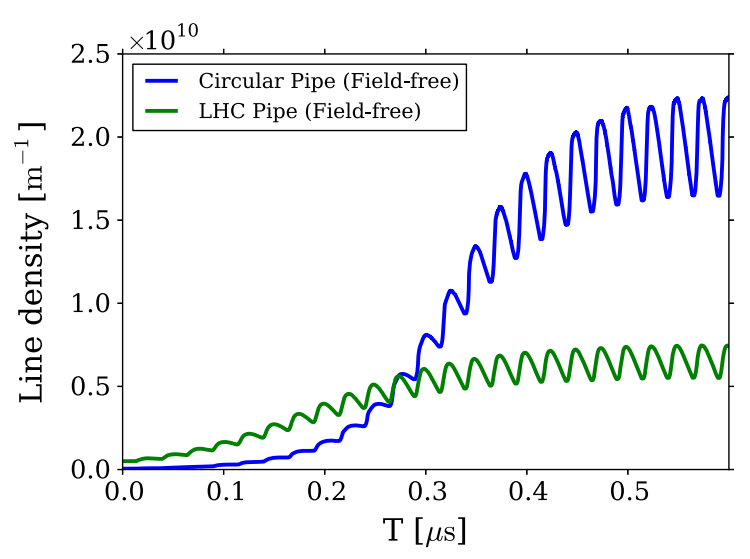

FIG. 18. Electron cloud buildup in the realistic LHC pipe geometry (green) and in the circular geometry (blue). Bunch population is $10^{11}$.
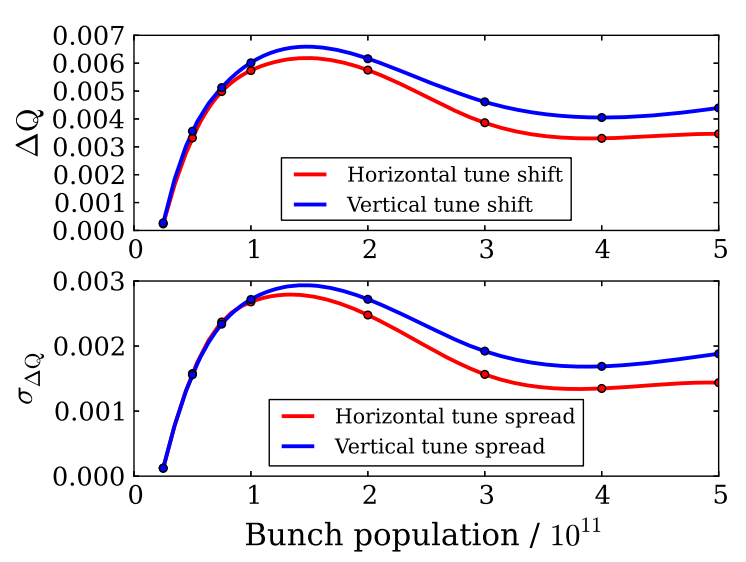

FIG. 19. Tune shifts (top) and tune spreads (bottom) as a function of bunch population for the LHC geometry. The result for horizontal (red) and vertical (blue) planes is shown.

diluted density region in the middle and a dense sheath near the wall as well as density stripes in the external magnetic field. The buildup curves for the realistic and circular geometries in the field-free section are shown in Fig. 18. For the LHC pipe geometry and without a magnetic field the electron cloud density is about 3 times lower than for the circular pipe. There are two possible reasons for this: fewer electrons are hitting the wall at exactly $W_{\mathrm{SEY} \text {,max }}$, or because of the missing SEY dependence on the angle of incidence is absent in our model. However, no significantly new features of the cloud density profile are observed. At the same time, in dipole field the density is larger and stays close to the case of circular geometry. The formation of the central stripe is also observed for higher intensities.

Figure 19 shows the horizontal and vertical tune shifts as a function of bunch population in the field-free region. For bunch populations below $10^{11}$ the tune shifts are similar and the bunch does not see the asymmetric shape of the cloud. For higher intensities a difference between two planes arises. In the studied intensity range we find
$\Delta Q_{x} / \Delta Q_{y} \approx h_{y} / R_{x} \approx 0.8$. This observation agrees with Eq. (18) if one assumes that the cloud is uniform and the LHC pipe is elliptic. However, contrary to Eq. (18) for $N_{i}<10^{11}$ the tune shifts in horizontal and vertical planes are similar. It is important to note that for the realistic pipe geometry in a dipole magnet a negative tune shift appears already for a bunch population of $1.5 \times 10^{11}$.

[1] F. Zimmermann, Phys. Rev. ST Accel. Beams 7, 124801 (2004).

[2] O. Dominguez, K. Li, G. Arduini, E. Metral, G. Rumolo, F. Zimmermann, and H.M. Cuna, Phys. Rev. ST Accel. Beams 16, 011003 (2013).

[3] G. Franchetti, I. Hofmann, W. Fischer, and F. Zimmermann, Phys. Rev. ST Accel. Beams 12, 124401 (2009).

[4] O. Boine-Frankenheim, E. Gjonaj, F. Petrov, F. Yaman, T. Weiland, and G. Rumolo, Phys. Rev. ST Accel. Beams 15, 054402 (2012).

[5] M. A. Furman and A. A. Zholents, in Proceedings of the 18th Particle Accelerator Conference, New York, 1999 (IEEE, New York, 1999), pp. 1794-1796.

[6] K. Ohmi et al., Reports No. SLAC-PUB-9079 and No. CERN-SL-2001-062-AP.

[7] T. Ieiri, J. Flanagan, H. Fukuma, Y. Ohnishi, and M. Tobiyama, in Proceedings of the International Particle Accelerator Conference, Kyoto, Japan (ICR, Kyoto, 2010), pp. 1934-1936.

[8] S. Ahmed, B. Yunn, J. Dolph, T. Satogata, and G. A. Krafft, in Proceedings of the 2nd International Particle Accelerator Conference, San Sebastián, Spain (EPS-AG, Spain, 2011), pp. 817-819.

[9] G. Dugan et al., in Proceedings of the 3rd International Particle Accelerator Conference, New Orleans, LA, 2012 (IEEE, Piscataway, NJ, 2012), pp. 2081-2083.

[10] H. Bartosik, W. Hoe, G. Iadarola, Y. Papaphilippou, and G. Rumolo, Benchmarking Headtail with Electron Cloud Instabilities Observed in the LHC, CERN Yellow Report CERN-2013-002 (CERN, Geneva, 2013), pp. 211-217.

[11] G. Arduini et al., in Proceedings of the 8th European Particle Accelerator Conference, Paris, 2002 (EPS-IGA and CERN, Geneva, 2002), pp. 1437-1439.

[12] M. T. F. Pivi et al., in Proceedings of the 11th European Particle Accelerator Conference, Genoa, 2008 (EPS-AG, Genoa, Italy, 2008), pp. 688-690.

[13] O. Haas, O. Boine-Frankenheim, and F. Petrov, Nucl. Instrum. Methods Phys. Res., Sect. A 729, 290 (2013).

[14] G. Rumolo and G. Iadarola, 5th RLIUP Meeting, 2013.

[15] F. Petrov and O. Boine-Frankenheim, Nucl. Instrum. Methods Phys. Res., Sect. A 723, 143 (2013).

[16] E. Benedetto, D. Schulte, F. Zimmermann, and G. Rumolo, Phys. Rev. ST Accel. Beams 8, 124402 (2005).

[17] S. Heifets, Qualitative Analysis of Electron Cloud Effects in the NLC Damping Ring, CERN Yellow Report CERN2002-001 (CERN, Geneva, 2002), pp. 129-136.

[18] J. S. Berg, LHC Project Note 97, 1997.

[19] X. S. Li, ACM Trans. Math. Softw. 31, 302 (2005).

[20] M. A. Furman and M. T. F. Pivi, Phys. Rev. ST Accel. Beams 5, 124404 (2002). 
[21] H. M. Cuna, J. G. Contreras, and F. Zimmermann, Phys. Rev. ST Accel. Beams 15, 051001 (2012).

[22] R. Cimino, I. Collins, M. Furman, M. Pivi, F. Ruggiero, G. Rumolo, and F. Zimmermann, Phys. Rev. Lett. 93, 014801 (2004).

[23] A. U. Luccio and N.L. D'Imperio, Eigenvalues of the One-turn Matrix, C-A/AP\#126, 2003.

[24] G. Iadarola et al., Electron Cloud in the LHC, Electron Cloud Meeting \#7, 2013, CERN.

[25] D. R. Grosso et al., in Proceedings of the 11th European Particle Accelerator Conference, Genoa, 2008 (Ref. [12]), pp. 1619-1621.
[26] M. A. Furman, R. De Maria, Y. Papaphilippou, and G. Rumolo, in Proceedings of the International Particle Accelerator Conference, Kyoto, Japan (Ref. [7]), pp. 1958-1960.

[27] M. A. Furman and V. H. Chaplin, Phys. Rev. ST Accel. Beams 9, 034403 (2006).

[28] E. Benedetto and F. Zimmermann, in Proceedings of the 9th European Particle Accelerator Conference, Lucerne, 2004 (EPS-AG, Lucerne, 2004), pp. 18341836.

[29] O. S. Brüning, LHC Project Report No. 158, Geneva, 1997. 